\title{
CHAPITRE 1 \\ Les réformes du droit français de la responsabilité civile
}

Denis Mazeaud

Professeur à Université Panthéon-Assas, Paris 2

1. Le ministère de la Justice a récemment décidé de réformer le droit des obligations. Cette réforme tant attendue se fera en deux temps. D'une part, en 2016, le droit des contrats sera réformé par voie d'ordonnance. D'autre part, dans un second temps, le droit de la responsabilité civile le sera par la voie parlementaire... Enfin, le législateur prête attention au droit des obligations, la belle endormie du Code civil, qui, pour l'essentiel, est demeuré inchangé depuis plus de deux siècles. L'annonce de cette réforme prochaine a d'autant plus surpris et réjoui la communauté des juristes français qu'elle était un peu l'Arlésienne du droit privé, depuis près de 10 ans, précisément depuis qu'en 2004, lors d'une des célébrations du bicentenaire du Code civil en Sorbonne, le président de la République de l'époque, Monsieur Jacques Chirac, avait lancé à la tribune : « Il faut réformer le droit des obligations ».

2. Depuis cet appel, règne une certaine effervescence qui s'est traduite par la réalisation de plusieurs avant-projets. C'est d'abord, en 2005, une armada de professeurs de droit qui, sous la houlette de Pierre Catala, a rédigé un avant-projet qui a pour ambition d'opérer un changement dans la continuité, sans rupture avec les grands principes qui irriguent le modèle français. Puis, en 2009, le Sénat a publié un rapport d'information intitulé "Responsabilité civile: des évolutions nécessaires». Cette initiative parlementaire comporte par toute une série de propositions plus ou moins innovantes et audacieuses en vue de réformer le droit de la responsabilité civile. Enfin, du moins si on s'en tient aux avant-projets officiellement publiés, un dernier a vu le jour, majoritairement conçu par des universitaires sous la direction de François Terré, soucieux que la réforme se traduise par une rupture avec les règles et les notions emblématiques de la responsabilité. 
Reste que, jusqu'au mois de novembre dernier, en dépit de ces nombreux avant-projets, aucune réforme ne se profilait à l'horizon: «toujours plus de projets, toujours moins de réforme!" s'amusaient certains, qui n'hésitaient pas d'ailleurs à émettre des doutes sur l'opportunité d'une réforme du Code civil, sans laquelle notre droit de la responsabilité évoluait malgré tout au rythme jurisprudentiel de l'évolution de notre environnement économique, technologique, scientifique, politique et social, et avec laquelle le risque de rigidification de notre droit était à redouter.

3. Il ne fait pourtant guère de doute à nos yeux que la réforme du droit de la responsabilité, s'impose, si tant est que l'on croit aux vertus de la codification, à savoir l'accessibilité, la prévisibilité et la stabilité du droit.

D'abord, parce qu'il existe un très embarrassant contraste entre le Code civil et le droit positif de la responsabilité. Brevitatis causa, le Code civil n'est plus, loin s'en faut le reflet fidèle et sincère du droit contemporain... Le Code civil a vieilli, et plutôt mal vieilli d'ailleurs, tant en ce qui concerne son esprit que sa lettre. Conçu à l'origine dans le Code de 1804, dont la lettre est pour l'essentiel restée inchangée si on excepte l'incorporation des règles sur la responsabilité du fait des produits défectueux, comme un droit destiné à régir les relations sociales en canalisant la liberté individuelle via la mise en jeu de la responsabilité civile de ceux qui causent fautivement un dommage à autrui, appréhendé il y a deux siècles comme un droit qui prévenait et réprimait les comportements fautifs dommageables, notre droit de la responsabilité civile tel que "gravé » dans le Code est vieilli, désuet, dépassé, inadapté. Sans compter que certaines de ses dispositions ont été purement et simplement abrogées par la Cour de cassation, par exemple la règle de la responsabilité pour faute présumée des parents du fait de leur enfant mineur, qui s'est muée en responsabilité sans faute. Le Code doit donc être sensiblement restauré et rénové, d'une part, pour réconcilier avec notre droit positif, en intégrant les règles énoncées par la jurisprudence pendant plus d'un siècle pour faire évoluer notre droit malgré son immobilisme, d'autre part, pour faire face aux nouveaux défis de notre société, que ce soit pour appréhender les risques anormalement dangereux provoqués par des activités industrielles ou commerciales, pour neutraliser les dommages de masse qui emportent des atteintes irréversibles, pour affronter les dommages qui menacent l'avenir des générations futures.

Ensuite, parce qu'en l'état notre droit de la responsabilité civile est relativement au moins inaccessible et, donc, imprévisible. Il est aujourd'hui, en effet, difficile pour le justiciable, voire le juriste, d'accéder au droit 
positif de la responsabilité civile dont notre Code ne donne qu'un très vague reflet. Depuis la première moitié du vingtième siècle, ce droit a évolué malgré l'immobilisme de la loi et ses mutations les plus profondes sont à mettre au crédit de la plume des juges qui ont progressivement substitué au droit classique de la responsabilité un droit moderne de l'indemnisation, relayé dans cette perspective par le législateur depuis le dernier quart du siècle dernier. Reste que notre droit positif est, en raison de cette édification par strates successives, un droit émietté, éparpillé dans le Code civil, d'autres codes (tel que celui de la santé publique), le Bulletin des arrêts de la Cour de cassation et le Journal officiel de la République française, étant entendu que la plupart des lois d'indemnisation contemporaines n'ont pas trouvé refuge dans le Code civil. Aussi, notre Code civil ne constitue-t-il plus le support du droit positif de la responsabilité civile, lequel a trouvé refuge dans d'autres codes ou recueils et dont il n'offre que quelques fragments, si ce n'est quelques vestiges; il n'est plus l'écrin du droit vivant.

Enfin, parce que seule une intervention législative permettra une réforme d'envergure dont notre droit a manifestement grand besoin. D'une part, pour inscrire dans le long terme et avec la précision requise, les évolutions nécessaires en vue d'une rénovation profonde et pérenne de notre droit de la responsabilité. D'autre part, pour opérer des arbitrages indispensables entre les tendances sociale qui prônent une sorte de droit naturel à l'indemnisation et proposent de retenir comme centre de gravité de ce droit les intérêts de la victime, et libérale qui dénoncent les dérives de la victimologie et prêchent pour un retour de l'idée de... responsabilité dans un droit aujourd'hui submergé par l'idéologie de la réparation, voire pour une économie de l'indemnisation.

4. Les raisons de la réforme étant désormais identifiées, du moins certaines d'entre elles, on peut s'arrêter brièvement sur les objectifs que le gouvernement a choisis, ou dont il serait opportun qu'il s'inspire pour que la révision du Code civil débouche sur une réforme du droit de la responsabilité civile, digne de ce nom.

Pour réaliser la réforme qui s'impose, le gouvernement devra accomplir une triple mission.

D'abord, une mise à jour de notre Code civil, laquelle s'impose si on veut que notre droit écrit redevienne le reflet de notre droit vivant. Les textes qui, dans celui-ci, concernent la responsabilité civile exigent une profonde refonte, du moins les articles historiques : certains ne reflètent plus le droit positif, on pense par exemple à l'article 1384, alinéa 7 , d'autres sont manifestement en sursis, on songe à l'article 1386, d'autres 
encore ont peut-être leur avenir prestigieux derrière eux, on ose « dénoncer » l'article 1384, alinéa $1^{\text {er }}$. Mais surtout, le Code est incomplet. D'une part, parce que, pour des raisons qui restent à éclaircir, certaines règles d'origine légale qui font partie intégrante de notre droit positif n'ont pas trouvé refuge en son sein, la loi du 9 juillet 1985 sur l'indemnisation des accidents de la circulation, entre autres. D'autre part, parce sont absentes $\mathrm{du}$ Code, toutes les règles de notre droit contemporain de la responsabilité civile qui ont été conçues par la Cour de cassation et qu'il convient désormais, d'y incorporer si on veut que notre droit écrit soit le reflet de notre droit vivant. En clair, il conviendra donc d'intégrer dans le Code civil toutes les règles du droit de la responsabilité civile qui régissent et qui charpentent celui-ci depuis plusieurs décennies, mais qui sont hors du Code. Bref, la première entreprise à laquelle le gouvernement doit s'atteler réside dans la codification du droit des contrats.

Ensuite, une mise en ordre de notre droit de la responsabilité civile est impérative. Les réformateurs devront le repenser de fond en comble pour lui redonner une cohérence, une unité et une intelligibilité dont son mode d'évolution, par strates législatives et jurisprudentielles successives, l'a privées. A l'occasion de cette réforme d'ensemble, il convient de remettre de l'ordre dans ce droit éclaté, de choisir quelques grandes idées directrices autour desquelles il puisse être harmonieusement articulé. Il faut substituer une toile, non seulement restaurée mais aussi renouvelée, une fresque harmonieuse à la mosaïque actuelle.

Enfin, une mise au point de notre droit de la responsabilité civile est nécessaire. Il appartiendra aux auteurs de la réforme de prendre parti sur des questions absolument fondamentales, en gardant à l'esprit que leur œuvre s'inscrira fatalement dans la durée et qu'ils doivent donc se projeter dans le long terme. Aussi, devront-ils décider, par exemple, si la notion de préjudice doit de distendre pour permettre la réparation du préjudice écologique pur, si le principe général de responsabilité du fait des choses doit céder la place à un principe de responsabilité du fait des activités anormalement dangereuses, s'il n'est pas temps d'admettre les dommages et intérêts punitifs, si le droit de la responsabilité doit continuer à être exclusivement conjugué au passé ou si il doit aussi permettre, à l'aide nouveaux outils comme le principe de précaution, d'appréhender les risques de dommages futurs gravissimes et irréversibles, etc.

5. Reste que, et c'est sans doute ce qui fait la singularité du droit français contemporain de la responsabilité civile, la complainte de l'absence de réforme doit être ramenée à sa juste proportion : en effet, l'absence de révision du Code civil, pendant toutes ces années, n'a pas rimé avec l'absence de réforme du droit. En effet, il n'est pas excessif de dire qu'en 
dépit de l'immobilisme du Code, notre droit de la responsabilité s'est purement et simplement métamorphosé grâce, essentiellement, à la plume audacieuse et inventive de la Cour de cassation. Métamorphose qui s'est concrètement traduite par la création de concepts et de règles qui ont marqué notre droit de leur très forte empreinte.

6. Pour esquisser concrètement ce que pourrait être la future réforme et en apprécier les vices et les vertus, on se livrera à un petit exercice comparatif dont les deux termes seront deux avant-projets de réforme précités : l'Avant-projet de réforme du droit des obligations et de la prescription $^{1}$, d'une part, dont les dispositions relatives à la responsabilité ont été élaborées par quelques professeurs d'université sous la direction de Madame Viney; l'avant-projet rédigé sous l'égide de l'Académie des sciences morales et politiques ${ }^{2}$, d'autre part ${ }^{3}$, dont les chefs de file ont été Messieurs Philippe Rémy et Jean-Sébastien Borghetti.

Cette comparaison est d'autant plus stimulante que ces deux avant-projets sont profondément distincts, à tel point qu'il n'est pas excessif d'affirmer que leur étude comparée révèle un véritable choc culturel et idéologique. Avec l'Avant-projet Catala, on s'inscrit dans la grande tradition française, tant les fondamentaux du modèle français sont déclinés avec classicisme, ce qui n'exclut pas quelques innovations tout à fait remarquables. En bref, l'essentiel de ce texte consiste à consacrer les acquis jurisprudentiels tout en modifiant à la marge le droit positif, une double œuvre de codification et de rénovation, donc...

Pour sa part, l'Avant-projet Terré propose rien moins qu'une véritable révolution culturelle, un changement de paradigme; il opte moins pour une consolidation du droit positif que pour une reconstruction, une révision de notre droit de la responsabilité civile, dont il modifie non seulement la lettre, mais aussi et surtout fondamentalement l'esprit. Ses promoteurs ont, en effet, mis en place " une politique civile qui tend à distinguer et à hiérarchiser les intérêts protégés $»^{4}$, et qui aboutit à substituer à l'idéologie de la réparation, une véritable économie de l'indemnisation, dont les objectifs consistent à «rationaliser la réparation des préjudices

1 Avant-projet qu'on citera dans la suite de notre texte, «Avant-projet Catala », du nom de celui qui l'a dirigé et qui a été publié à la documentation française (2006).

2 Avant-projet qu'on citera dans la suite de notre texte, "Avant-projet Terré », du nom de celui qui l'a dirigé et qui a été publié aux éditions Dalloz: Pour une réforme du droit de la responsabilité civile, Dalloz, 2011.

3 On exclura de cette comparaison, l'avant-projet récemment préparé par le ministère de la Justice, encore officieux et secret à l'heure où ces lignes sont écrites.

4 Ph. Rémy et J.-S. Borghetti, «Présentation du projet de réforme de la responsabilité délictuelle », in Pour une réforme du droit de la responsabilité civile, Dalloz, 2011, sp. $n^{\circ} 8$. 
résultant d'une atteinte à la personne, limiter la réparabilité des préjudices réfléchis, régler aussi clairement qu'il est possible la réparation des préjudices moraux $»^{5}$.

Pour que le contraste culturel, politique et technique entre les deux avant-projets comparés, ressorte avec le plus de netteté possible, on se livrera à des comparaisons relatives à leurs esprits (I) leurs structures (II) et à leurs lettres (III) respectifs.

\section{L'esprit}

7. Pendant près d'un siècle, notre droit de la responsabilité a évolué malgré l'inertie du législateur et l'immobilisme du Code. Même si la fin du vingtième siècle a été marqué par un retour en force de la loi ${ }^{6}$, il n'en reste pas moins qu'en matière de responsabilité civile, c'est le juge qui a créé les principes généraux, alors que le législateur s'est attelé aux régimes spéciaux d'indemnisation; autrement dit, la Cour de cassation a fixé les grandes vues, tandis que la loi s'est plutôt attachée aux cas particuliers ${ }^{7}$

L'Avant-projet Catala poursuit dans une certaine mesure cette coopération des forces créatrices du droit de la responsabilité en accordant une marge certaine de manœuvre au juge, lequel n'est donc pas réduit au rang peu glorieux de simple bouche de la loi et est appréhendé comme une sorte de coproducteur dans les productions des normes.

Pour sa part, l'Avant-projet Terré adopte, quant aux sources du droit de la responsabilité, un parti très sensiblement différent. Selon ses auteurs, c'est au législateur de faire les choix les plus importants, de montrer la direction et de fixer le cap : "la stabilité du système et la prévisibilité des solutions y gagneraient, au moins pour un temps ${ }^{8}$; aussi, "dès lors que le législateur reprend la main en recodifiant le droit de la responsabilité civile, il n'y a pas de raison de conserver au juge le pouvoir qu'il avait acquis ${ }^{9}$. Ce changement dans la répartition des forces créatrices reflète une mutation politique forte: il s'agit de lutter contre l'idéologie de la réparation qui anime le juge depuis des décennies, pour édifier une économie de l'indemnisation, et c'est alors "au législateur de sélectionner les intérêts qu'il entend protéger, contre quelles atteintes et

\footnotetext{
5 Ibid, sp. $n^{\circ} 15$.

6 Loi d'indemnisation des victimes d'accidents de la circulation (1985) et sur la responsabilité du fait des produits défectueux (1998).

7 Ibid, sp. n 2.

8 Ibid, sp. $\mathrm{n}^{\circ} 4$.

9 Sp. $n^{\circ} 7$.
} 
à quelles conditions ${ }^{10}$. Cette mutation radicale se traduit, notamment, dans l'Avant-projet Terré par les règles qui disposent que le législateur a le monopole de la création des cas de responsabilité sans faute et du fait d'autrui : les régimes de responsabilité sans faute ou du fait d'autrui sont donc d'origine légale ou ne sont pas... Avec de telles dispositions, à l'avenir, plus d'arrêt Teffaine ou Jand'heur, pas d'arrêt Blieck, etc.

8. La dépendance du juge dans l'Avant-projet Terré existe aussi, au stade de l'indemnisation du préjudice, à l'égard du pouvoir réglementaire. Dans la perspective de la rationalisation de la réparation des préjudices extrapatrimoniaux, les concepteurs de ce texte ont succombé aux charmes de la barêmisation édifiée par voie réglementaire pour l'indemnisation des préjudices corporels. Dans ce domaine, je juge est donc lié ; fini le sur-mesure, place au prêt-à-porter imposé. On peut se demander si cette défiance à l'égard du juge ne se traduira pas fatalement par une atteinte sensible au principe de la réparation intégrale.

Quant à lui, l'Avant-projet Catala n'exclut pas les barèmes, mais il y sacrifie beaucoup moins aveuglément puisqu'il les cantonne au seul préjudice fonctionnel.

9. Autre différence fondamentale qui affecte l'esprit des deux avant-projets comparés, le sort dévolu par chacun aux clauses générales.

Fort classiquement, l'Avant-projet Catala est resté fidèle à la tradition française des clauses générales, que l'on retrouve énoncées tant en matière de faute que de fait des choses et de fait d'autrui, les deux dernières ayant été crées par la Cour de cassation. Cet attachement à cette construction tient à ce qu'elle offre le double avantage de la plasticité et de l'adaptabilité. Ces clauses générales permettent, en effet, au juge d'appréhender les dommages de toutes sortes, nouveaux, imprévus, inattendus, inimaginables, originaux, etc. On relèvera que le principe général de responsabilité du fait des choses est officiellement introduit par ce texte dans le Code, avec le régime tel qu'il a été ciselé par la Cour de cassation au siècle dernier (présomption de garde pesant sur le propriétaire de la chose, présomption de responsabilité pesant sur le gardien, exonération par la force majeure).

L'Avant-projet Terré fait, au contraire, montre de méfiance à l'égard de ce type de clauses, parce que leur généralité "n'offre structurellement aucune résistance aux demandes de réparation, lesquelles sont abandonnées à la prudence des juges (à leur bon cour) » ${ }^{11}$. Cette méfiance

10 Sp. $n^{\circ} 10$.

11 Ph. Rémy, «Réflexions préliminaires sur le chapitre des délits », Pour une réforme du droit de la responsabilité civile, préc., sp. p. 39. 
se traduit par le maintien d'une seule clause générale, la responsabilité pour faute, et surtout par la disqualification de la responsabilité du fait des choses qui est reléguée au rang de simple délit spécial, à l'instar des troubles anormaux du voisinage, du fait des produits défectueux ou du fait des véhicules terrestres à moteur... " Pire ", le délit spécial du fait des choses se voit assigné un domaine strict, puisqu'il est circonscrit aux seuls dommages corporels causés par une chose corporelle, et soumis à un régime rigoureux, étant entendu que la victime doit toujours supporter la charge, et donc le risque, de la preuve du fait actif de la chose, alors qu' elle en est parfois dispensée en droit positif, notamment lorsqu'elle est entrée en contact avec une chose en mouvement.

\section{La structure}

10. Sur un plan structurel, aussi, les deux avant-projets connaissent de sensibles différences, notamment quant au traitement réservé par ces textes à la distinction entre les deux ordres de responsabilité.

Abandonnant la structure retenue par les rédacteurs du Code civil, l'Avant-projet Catala regroupe sous un titre unique les règles qui régissent les responsabilités contractuelle et délictuelle, au motif que «les règles communes aux deux branches de la responsabilité l'emportent très largement $\gg^{12}$ sur les règles qui leurs sont spécifiques. Ce faisant, les auteurs de cet avant-projet prennent implicitement, mais clairement, parti sur le concept même de responsabilité contractuelle; ils ne se rangent pas à la position de ceux qui considèrent que les dommages-intérêts contractuels ne relèvent pas de l'idée de responsabilité et ne constituent qu'une simple modalité de l'exécution de l'obligation contractuelle. Dans l'Avant-projet Catala, la responsabilité contractuelle existe bel et bien, et elle entretient un certain degré de parenté avec la responsabilité délictuelle.

On remarquera aussi que cet avant-projet maintient la fameuse règle du non-cumul, qui traduit la hiérarchie entre les deux ordres de responsabilité. En droit positif, la responsabilité délictuelle constitue, en effet, le principe et la responsabilité contractuelle, l'exception, laquelle n'a vocation à régir que l'indemnisation des seuls dommages causés par un manquement à une obligation contractuelle imputable au débiteur et subis au cours de l'exécution d'un contrat par le créancier. D'où les règles traditionnelles selon lesquelles un contractant victime d'un

12 G. Viney, "Exposé des motifs", in Avant-projet de réforme du droit des obligations et de la prescription, préc., sp. p. 162. 
dommage contractuel, quand il entre dans le champ de la responsabilité contractuelle, ne peut ni opter, alors même qu'il y aurait intérêt, pour les règles de la responsabilité extracontractuelle, ni combiner les règles contractuelles et extracontractuelles. Inversement, un tiers victime d'un dommage causé par un manquement contractuel ne peut exercer qu'une action en responsabilité extracontractuelle contre le débiteur qui lui a causé son préjudice. Le texte apporte des tempéraments absolument remarquable à ces principes qui tracent la frontière entre les deux ordres de responsabilité : en premier lieu, le créancier victime d'un préjudice corporel peut, s'il y a intérêt, exercer une action en responsabilité délictuelle; en second lieu, le tiers victime d'un dommage causé par le manquement d'un débiteur contractuel peut exercer une action contractuelle contre celui-ci, lequel pourra alors l'attraire dans son contrat et lui opposer tous les moyens dont il aurait pu se prévaloir contre son propre contractant.

11. Toute autre est la position adoptée par les concepteurs de l'Avant-projet Terré! Parce que ceux-ci nient le concept même de responsabilité contractuelle, ils excluent purement et simplement cette « responsabilité » de leur texte et s'inscrivent, non seulement dans le droit fil du Code civil, qui intégrait la question des dommages-intérêts contractuels dans la subdivision relative au contrat, mais encore dans tous les projets d'harmonisation européenne du droit des contrats, dans lesquels la responsabilité contractuelle est envisagée comme une troisième voie, un troisième remède offert au créancier contractuel insatisfait, avec l'exécution forcée en nature et la résolution du contrat inexécuté. Les fonctions distinctes de la responsabilité délictuelle (réparer le dommage injustement causé à autrui) et de la responsabilité contractuelle (fournir au créancier l'équivalent de l'avantage escompté du contrat) emportent cette distinction structurelle et substantielle fondamentale. Dans le prolongement de cette distinction essentielle opérée dans l'Avant-projet Terré, la responsabilité délictuelle reçoit une compétence exclusive pour la réparation des préjudices corporels, y compris ceux qui procèdent de l'inexécution d'un contrat. Exit alors les obligations de sécurité que le juge a successivement découvertes dans la majorité des contrats spéciaux depuis des décennies pour compléter le contenu du lien contractuel, sur le fondement de l'article 1135, et que l'Avant-projet Catala a, quant à lui, consacrées! Le texte trace une frontière idéologique très nette entre ce qui relève de l'être et de l'avoir, du corps de la victime et de son patrimoine: "les bras cassés et les morts d'homme " relèvent exclusivement de la responsabilité délictuelle, alors même qu'ils sont survenus à l'occasion de la mauvaise exécution d'un contrat, dont le centre de gravité réside dans la prestation d'ordre économique que sa création 
emporte à la charge des contractants, laquelle a l'exclusivité de l'application des règles contractuelles.

12. Autre différence structurelle remarquable, celle qui affecte la responsabilité du fait d'autrui.

L'Avant-projet Terré se singularise ainsi par une vision originale, en effet alors que, depuis des lustres, la responsabilité du fait d'autrui est appréhendée au même titre que le fait personnel ou le fait de la chose, comme un fait générateur de responsabilité, l'avant-projet la dissocie de ces derniers et traite des cas de responsabilité du fait d'autrui dans une subdivision intitulée « Du délit civil en général » à la suite de la faute, du dommage et de la causalité, sous le titre "De l'imputation du dommage causé par autrui ». Nul doute que cette architecture déroutera car elle va au rebours de la présentation traditionnelle et unanime en doctrine. Pourtant, cette innovation se justifie assez aisément car la spécificité de la responsabilité du fait d'autrui ne tient pas à celle de son fait générateur, mais à la manière dont la charge de la réparation est imputée. $\mathrm{Si}$ la responsabilité du fait d'autrui se distingue des autres responsabilités, c'est qu'elle pèse, non pas sur celui qui a "commis " le fait générateur, mais sur une autre personne, en vertu du lien que celle-ci entretient avec le responsable « naturel ». Ce n'est donc pas le fait générateur qui change, mais l'identité de celui sur qui pèse l'obligation de réparer le dommage causé par ce fait générateur, et l'imputation est justement ce qui permet de faire le lien entre le fait générateur et le débiteur. Cela explique que le projet traite de la responsabilité du fait d'autrui à part des autres «cas particuliers » de responsabilité.

\section{La lettre}

13. L'examen des dispositions des deux avant-projets comparés révèle que l'un et l'autre se sont livrés, quoique dans une mesure différente, à une double entreprise de codification $(\mathrm{A})$ et de modification $(\mathrm{B}) \mathrm{du}$ droit positif.

\section{A. Codification}

L'examen comparé de la codification à laquelle les deux avant-projets ont opportunément sacrifié fait apparaître des convergences (1) et des divergences (2). 


\section{1) Convergences}

14. A propos de la responsabilité du fait personnel, d'abord, on relèvera que la faute de la victime inconsciente ne peut plus, à l'inverse de la solution inéquitable du droit positif, diminuer le montant de son indemnisation. Depuis la loi du 3 janvier 1968 et les arrêts rendus en 1984 par la Cour de cassation ${ }^{13}$, la responsabilité des inconscients peut être engagée pour les dommages que ceux-ci causent à des tiers. Cette responsabilité, favorable aux victimes, a été rendue possible en raison de l'émancipation du concept même de responsabilité par rapport à l'idée de liberté, de libre arbitre, de culpabilité: les inconscients, infantes et déments, sont responsables mais pas coupables, et pour retenir cette responsabilité des « innocents " a été imaginé le concept fort ingénieux de faute objective. Faute débarrassée de toute connotation morale, déconnectée de la condition d'imputabilité, qui se réduit à un fait objectivement illicite un point c'est tout, quel que soit l'état de l'agent qui l'a commise. Fatalement, cette création prétorienne, consacrée dans les deux avant-projets, s'est retournée contre les inconscients : retenue pour mettre en jeu leur responsabilité, la faute objective doit, inversement, mécaniquement et fatalement, pouvoir être retenue pour diminuer le montant de l'indemnisation des dommages qu'ils subissent et à la réalisation duquel ils ont « fautivement » contribué. Véritable injure à l'équité, la règle du droit positif est donc écartée par les deux avant-projets : la faute objective d'une victime inconsciente ne peut plus lui être opposée pour réduire son droit à indemnisation.

15. Dans le domaine de la responsabilité du fait d'autrui, on relèvera, d'une part, qu'un frein est posé, dans les deux textes, à l'aggravation de la responsabilité des parents d'un mineur qui a causé un dommage. Certes, contrairement à la lettre du Code civil et conformément à un arrêt rendu en 1997 par la Cour de cassation, la responsabilité des parents restera une responsabilité sans faute. Mais les deux avant-projets sont moins sévères que l'est devenue la Cour de cassation qui, décide qu'il suffit, pour que leur responsabilité objective puisse être engagée, que le fait de l'enfant ait causé le dommage, indépendamment du point de savoir si ce fait dommageable est susceptible d'emporter la mise en jeu de sa responsabilité personnelle. En droit positif, les parents sont donc moins responsables des dommages causés par leurs enfants, que garants des préjudices subis par les victimes de ceux-ci. Les deux avant-projets rompent heureusement avec cette jurisprudence, qui repose sur l'existence de l'assurance souscrite par les parents, mais qui rompt, dans une mesure certaine, avec

13 Cass. ass. plén., 13 déc. 2002 : D. 2003, 231, note P. Jourdain ; Gaz. Pal. 2003, 52, note F. Chabas; JCP 2003.I.154, obs. G. Viney. 
l'idée même de responsabilité. Pour que la responsabilité des parents du fait dommageable de leur enfant mineur puisse être mise en jeu, il faudra nécessairement que la responsabilité personnelle de celui-ci puisse aussi être engagée. Ainsi, la responsabilité parentale redeviendra une véritable responsabilité du fait d'autrui, à savoir une responsabilité qui s'ajoute à la responsabilité personnelle de l'auteur du dommage et qui est conçue comme une garantie pour les victimes contre l'insolvabilité d'autrui, et plus comme une responsabilité exclusive et de substitution dont l'auteur direct (autrui) profite illégitimement.

16. Toujours en matière de responsabilité du fait d'autrui, il convient, en second lieu, de s'arrêter sur la consécration, dans les deux textes comparés, de la jurisprudence inaugurée par l'Assemblée plénière, le 29 mars 1991, dans son fameux arrêt «Blieck $»^{14}$. Par cet arrêt, la Cour de cassation a fait sensation puisqu'elle a créé de toutes pièces un nouveau cas de responsabilité du fait d'autrui, voire, selon certains, un principe général de responsabilité du fait d'autrui, réplique du principe général de responsabilité du fait des choses qu'elle avait créée en 1930. En substance, en 1991, la Cour de cassation a énoncé une règle de responsabilité, pour les dommages causés par autrui, qui pèse sur les personnes qui organisent, dirigent et contrôlent le mode de vie d'autrui. La nature et le régime de la règle prétorienne, tels qu'ils ont été déterminés par la jurisprudence depuis plus de vingt ans, sont consolidés dans les deux avant-projets. Ainsi, à l'image de tous les autres cas de responsabilité du fait d'autrui, la responsabilité de ces personnes chargées judiciairement, administrativement ou conventionnellement de régler le mode de vie d'autrui suppose pour être mise en jeu que le fait d'autrui soit susceptible d'engager sa responsabilité personnelle. En outre, pour échapper à sa responsabilité, le répondant devra apporter la preuve que le dommage causé par la personne dont il devait régler le mode de vie a été causé par un événement constitutif de cause étrangère, doté des caractères de la force majeure. C'est donc bien d'une responsabilité sans faute du fait d'autrui dont il s'agit.

\section{2) Divergences}

17. D'importantes divergences apparaissent ainsi dans la codification des règles de responsabilité du fait d'autrui.

La portée de l'immunité du préposé, dont le principe a été énoncé par un arrêt de la Cour de cassation rendu le 25 février 2000, est sensiblement différente dans les deux textes. Favorable au préposé, puisqu'originellement conçue comme une responsabilité de complément destinée 
à garantir la victime contre l'insolvabilité du préposé, la responsabilité du commettant est devenue une responsabilité pesant exclusivement sur celui-ci, la règle du droit positif peut se révéler défavorable aux victimes dans l'hypothèse, qui n'est pas d'école, dans laquelle le commettant est dans l'incapacité de l'indemniser. D'où, l'exclusion de l'immunité du préposé dans l'Avant-projet Catala si le commettant est insolvable et si son assureur ne peut pas être appelé en garantie ${ }^{15}$, que ne retient pas l'Avant-projet Terré. Ainsi, dans l'Avant-projet Catala, l'immunité du préposé ne peut plus emporter une absence d'indemnisation pour les victimes; elle redevient une responsabilité subsidiaire par rapport à la responsabilité du commettant.

On remarquera aussi que la définition de l'abus de fonctions de ce même préposé est différente dans les deux textes. L'Avant-projet Catala reprend à son compte la définition retenue en dernier lieu par la Cour de cassation, dont on sait qu'il lui a fallu pas moins de cinq arrêts rendus soit en Chambres réunies, soit en Assemblée plénière, pour que, tiraillée entre l'impératif d'indemnisation des victimes, lequel suppose que le commettant ne puisse pas échapper trop aisément à sa responsabilité en se prévalant d'un abus de fonctions de son préposé, et un souci de justice élémentaire pour celui-là, qui impose que sa responsabilité ne puisse pas être retenue dans les hypothèses où son préposé a causé un dommage indépendant de ses fonctions, la Cour de cassation donne une définition satisfaisante de l'abus de fonctions. Définition équilibrée, en ce sens qu'elle paraît ménager les intérêts bien compris des uns et des autres, donnée le 19 mai 1988 et ainsi ciselée : «(...) le commettant ne s'exonère de sa responsabilité que si le préposé a agi hors de ses fonctions auxquelles il était employé, sans autorisation, et à des fins étrangères à ses attributions». C'est cette formule qui est reprise à la lettre par l'Avant-projet Catala, dans son article 1359, alinéa 2 , et complétée par une règle qui, elle aussi, consacre la jurisprudence de la Cour de cassation. En effet, dans ce même texte, la responsabilité du commettant est exclue lorsque celui-ci apporte la preuve que la victime du dommage commis par le préposé ne pouvait pas légitimement croire que ce dernier avait agi pour le compte du commettant. Règle inspirée par plusieurs arrêts rendus par la Cour de cassation, lorsque la victime est cocontractant du commettant, et que l'exécution du contrat a été " déléguée » au préposé.

L'Avant-projet Terré retient, pour sa part, une définition allégée de l'abus de fonctions du préposé, puisque l'employeur échappe à sa responsabilité s'il prouve que "le salarié a agi sans autorisation et à des fins étrangères

15 Etant précisé que l'Avant-projet Terré distingue la responsabilité sans faute de l'employeur du fait de son salarié, dont il est ici question, de la responsabilité pour faute du commettant du fait de son préposé. 
à son emploi ». En n'exigeant plus que l'employeur démontre que le salarié a agi hors de ses fonctions, les auteurs de ce texte espèrent que le juge ne se contentera plus d'un simple lien objectif entre le fait dommageable et les fonctions pour exclure l'abus de fonctions, et qu'ainsi des décisions excessivement rigoureuses pour l'employeur n'aient plus droit de cité dans notre droit futur. En effet, en application de la définition teintée d'une objectivité certaine, que la Cour de cassation a finalement retenue quant au lien entre les fonctions et le dommage, elle décide aujourd'hui qu'il suffit, pour que la responsabilité du commettant soit mise en jeu, que le préposé ait trouvé dans son emploi l'occasion et les moyens de sa faute. Avec la définition plus subjective retenue par l'Avant-projet Terré, ce type de solutions rigoureuses pour l'employeur est voué à disparaître.

18. On retiendra, enfin, que le statut du principe de la réparation intégrale diffère selon les avant-projets.

Alors que l'Avant-projet Catala réaffirme la force de ce principe avec la même vigueur (de façade ?) que la Cour de cassation, l'Avant-projet Terré énonce que la victime peut « en général »... demander la réparation intégrale de son préjudice... La nuance est de taille, assurément. Principe cardinal de notre système d'indemnisation, en vertu duquel la victime d'un préjudice doit recevoir une indemnisation intégrale du préjudice qu'elle a subi, pas plus et pas moins (tout le préjudice, mais rien que le préjudice), son insertion dans le Code civil réalisé par l'Avant-projet Catala est d'autant plus remarquable que l'on souligne assez souvent aujourd'hui en doctrine sa relativité, sa valeur incertaine, sinon l'artifice qui le fragilise en raison de la part importante laissée à l'appréciation souveraine du juge en cette matière spécifique. Autant de critiques qu'acte manifestement l'Avant-projet Terré avec sa formule laconique...

\section{B. Modification}

L'examen comparé des entreprises de modification du droit positif et donc d'innovation, fait apparaître, lui aussi, des divergences (1) et des convergences (2).

\section{1) Divergences}

19. Au rang des divergences, dans cet exercice d'innovations auquel se livrent les deux avant-projets, on s'arrêtera, une fois encore, sur quelques points symptomatiques.

En particulier, la responsabilité du fait d'autrui fait l'objet de traitements très sensiblement distincts.

Pour sa part, l'Avant-projet Catala crée de nouveaux cas de responsabilité du fait d'autrui : responsabilité du fait des personnes en situation 
de dépendance économique, d'une part, responsabilité des sociétés mères du fait de leurs filiales, d'autre part. Innovations remarquables et remarquées qui élargissent très sensiblement le champ des cas de responsabilité du fait d'autrui et reposent sur l'idée que la personne qui tire un profit économique de l'activité exercée par une autre, dont il organise et contrôle l'activité, peut voir sa responsabilité engagée à raison des dommages causés dans l'exercice de celle-ci. Avec ces nouveaux cas qui, concrètement, concernent les établissements de soins pour les dommages causés par leurs médecins salariés, les franchiseurs et concédants pour ceux provoqués par leurs distributeurs et, même, les sociétés mères pour les préjudices causés par leurs filiales, la responsabilité du fait d'autrui change de visage. Le critère de l'autorité sur autrui cède alors la place à celui de l'avantage économique tiré de l'activité sur autrui, et la simple existence d'une dépendance économique de l'auteur du dommage par rapport à son répondant est substituée à l'exigence de dépendance juridique entre l'un et l'autre.

Pour sa part, L'Avant-projet Terré opère, en effet, un changement tout à fait remarquable dans le régime dont est dotée la responsabilité des commettants. En effet, son article 17 substitue aux termes de commettant et de préposé ceux d'employeur et de salarié. Certes, on retrouve les termes classiques du Code civil dans l'article 18, mais alors la responsabilité du commettant est à cent lieux de celle du Code civil, puisqu'il s'agit d'une banale responsabilité pour faute du fait du préposé, lequel ne bénéficie alors d'aucune immunité de principe. L'Avant-projet Terré opère donc une distinction entre la responsabilité sans faute de l'employeur du fait de son salarié, d'une part, et la responsabilité pour faute présumée du commettant du fait de son préposé, d'autre part.

On pressent alors le recul de l'impératif d'indemnisation des victimes et la faveur faite aux commettants, autre qu'un employeur, qu'emporte cet article 18 de l'avant-projet qui, dans tous les cas où le lien de préposition n'est pas le fruit d'un contrat de travail, édicte un principe de responsabilité pour faute présumée. Recul sensible donc par rapport au droit positif, puisqu'aujourd'hui, tous les cas qui entrent dans le champ d'application de la responsabilité du fait d'autrui relèvent de la responsabilité objective. Ce qui couvre non seulement les hypothèses dans lesquelles le commettant et le préposé sont unis par un contrat de travail, mais encore celles dans lesquelles le répondant exerce une autorité sur autrui via une autre espèce de contrat ou en vertu d'un pouvoir de fait, ou encore exerce un contrôle sur l'activité exercée pour son compte et dans son intérêt par autrui. Désormais, et on pressent l'ampleur de la modification opérée, dans tous ces cas, la victime ne "bénéficiera " plus d'une responsabilité sans faute, qui n'offre au commettant que la seule échappatoire de la force majeure, celui-ci pourra s'exonérer en démontrant qu'il n'a pas commis 
de faute. La primauté des intérêts du commettant sur ceux de la victime est ainsi clairement affichée. En témoigne aussi l'absence de la moindre règle de responsabilité du fait d'autrui fondée sur le contrôle de l'activité économique exercée par autrui pour le compte et dans l'intérêt du répondant, en l'absence de dépendance juridique entre l'un et l'autre, qu'a retenue l'Avant-projet Catala. Illustration, parmi d'autres, de l'empreinte libérale de cet avant-projet.

20. Quant à la sélection des préjudices réparables, on s'arrêtera sur quelques exemples significatifs de la différence d'approche des deux textes comparés.

D'une part, en matière de préjudice environnemental, l'article 1343 de l'Avant-projet Catala prévoit l'indemnisation du préjudice consistant dans la lésion d'un intérêt " collectif », étant entendu, comme le précisent les auteurs du texte eux-mêmes, que ce terme a été introduit dans le texte "afin de permettre aux tribunaux d'admettre l'indemnisation du préjudice écologique ". L'article 8, alinéa 2, de l'Avant-projet Terré précise, en revanche, que "L'atteinte (...) à l'environnement est réparable dans les cas et aux conditions déterminées par la loi »; il s'agit alors de "prévenir des mouvements jurisprudentiels " ${ }^{16}$, incités par l'activisme environnemental de certaines associations, qui admettraient trop aisément l'indemnisation de préjudice écologique pur.

D'autre part, autre divergence remarquable, le traitement de la réparation du préjudice moral, dont nombreux sont ceux qui considèrent qu'elle présente parfois un caractère immoral. L'histoire est assurément un perpétuel recommencement puisqu'au début du XXème la controverse faisait rage en doctrine entre ceux qui considéraient qu'il est indigne de battre monnaie de ses larmes et ceux qui considèrent que le préjudice moral est un préjudice comme les autres, qui en tant que tel mérite donc d'être réparé. Si aujourd'hui, le débat rebondit, c'est parce que la réparation du préjudice moral a donné lieu à des dérives et qu'elle est l'objet d'une fuite en avant qui inquiètent même ceux qui ne s'opposent pas à son principe.

Alors que l'Avant-projet Catala pose le principe de la réparation du préjudice moral, l'A vant-projet Terré limite son admission : ainsi, la réparation du préjudice d'affection résultant de l'atteinte aux biens est exclue, sauf en cas de dommage intentionnel et de trouble grave subi par la victime $\mathrm{e}^{17}$. Il faut ajouter que le principe de la réparation intégrale du préjudice résultant d'une atteinte à l'intégrité morale est exclu ; pour de tels

16 Ph. Rémy et J.-S. Borghetti, préc., sp. $n^{\circ} 11$.

17 Art. 67. 
dommages, la réparation peut n'avoir qu'une portée symbolique ${ }^{18} \ldots \mathrm{Au}-$ tant dire que les auteurs de ce texte se rangent aux côtés de ceux qui ont, dès l'origine, trouvé qu'il n'était pas sain de battre monnaie de ses larmes.

Outre au sein des préjudices, contrairement à l'Avant-projet Catala, l'Avant-projet Terré opère aussi une sélection entre les victimes. Ainsi, les victimes par ricochet ne peuvent obtenir l'indemnisation de leur préjudice qu'à la condition que la victime directe ait subi un dommage résultant d'une atteinte à son intégrité corporelle ${ }^{19}$; en outre, seul son conjoint, ses parents, ses enfants et ses cohabitants pourront être indemnisés de leur préjudice d'affection, tandis que seuls ceux qu'elle entretenait pourront agir en réparation de leur préjudice économique. On ajoutera que les personnes morales ne peuvent obtenir la réparation de leur préjudice moral que si elles peuvent apporter la preuve que la cause de leur dommage réside dans une faute grave ${ }^{20}$.

21. Ultime divergence entre les deux avant-projets comparés, la question de l'étendue de la réparation du préjudice corporel. Dans l'Avant-projet Catala, seule une faute grave de la victime est susceptible de diminuer l'indemnisation de son préjudice corporel, faveur faite à la victime dont on ne trouve pas trace dans l'Avant-projet Terré. Nouveau tempérament apporté au principe de l'influence de la faute de la victime sur son droit à réparation, qui s'inscrit dans une des tendances lourdes de notre droit contemporain de l'indemnisation. Nouvelle faveur accordée aux victimes de dommages corporels qui doivent, dans l'idéologie de la réparation qui domine, toujours recevoir une réparation intégrale de ce type de préjudices.

\section{2) Convergences}

22. Au rang des innovations qui se caractérise par des convergences entre les deux avant-projets, illustration de leur esprit d'ouverture, l'admission de l'obligation imposée à la victime de modérer son dommage, obligation en vertu de laquelle, lors de l'évaluation de la créance de réparation, il est tenu compte des efforts consentis par la victime pour, sinon éviter, du moins diminuer sa perte. Dans les deux textes, le principe d'une telle obligation est retenu et il est doté d'un domaine et d'un régime identiques. Ainsi, les dommages corporels sont exclus du champ d'application de cette obligation et leur réparation ne peut donc pas être affectée par le comportement de la victime; de plus, il ne peut

18 Art. 69, al. 1er.

19 Art. 63, al. 1er.

20 Art. 68. 
être reproché à la victime de ne pas avoir agi pour diminuer le quantum de son préjudice que si elle pouvait le faire en prenant des mesures sûres, raisonnables et proportionnées. L'intégration de l'obligation de modérer le dommage dans notre droit est remarquable, à plusieurs titres... D'abord, parce qu'elle prend à rebours la Cour de cassation qui refuse toujours d'intégrer l'obligation de la victime de modérer son dommage en droit français. Ensuite, parce que cette obligation, que l'impératif d'efficacité économique du droit justifie parfaitement, dans la mesure où la réparation d'un dommage, lorsqu'on peut raisonnablement l'éviter ou le canaliser, est économiquement critiquable, heurte a priori de façon frontale le principe de la réparation intégrale. Autant dire que l'incorporation du devoir de minimiser son dommage ne va pas de soi, car la dimension morale du modèle français s'accommode mal de cette victoire de la loi du marché, de cette primauté de l' "économique " sur l'« éthique ». Pourtant dans l'exposé des motifs de l'Avant-projet Catala, Geneviève Viney justifie cette concession à la loi du marché en affirmant qu'elle "répond au souci de responsabiliser les victimes ${ }^{21}$. On comprend alors que, plus que de sacrifier aux délices de l'analyse économique du droit, il s'agit plutôt de moraliser le droit français de la réparation du dommage... L'ouverture d'esprit dont témoigne l'incorporation de cette règle étrangère à notre tradition ne rime donc pas avec l'abandon en rase campagne des valeurs fondamentales qui irriguent le modèle français de responsabilité civile.

23. Autre convergence notable entre les deux avant-projets, l'admission de dommages-intérêts autres que compensatoires, mais les deux textes diffèrent sur la nature de ces dommages-intérêts non compensatoires. Alors que l'Avant-projet Terré retient les seuls dommages-intérêts restitutoires, l'Avant-projet Catala ouvre les portes du Code civil aux dommages-intérêts punitifs. Aux termes de l'article 54 du premier d'entre eux, l'auteur d'une faute intentionnelle lucrative, celle qui permet à l'auteur d'un dommage de tirer un plus grand profit en le causant qu'en l'évitant, peut être obligé par le juge, à restituer à la victime le profit qu'il en a retiré, plutôt que l'équivalent de la perte subie par celle-ci. L'Avant-projet Catala est plus audacieux encore puisqu'il admet, dans son article 1371 que "L'auteur d'une faute manifestement délibérée, et notamment d'une faute lucrative, peut être condamné, outre les dommages-intérêts compensatoires, à des dommages-intérêts punitifs dont le juge a la faculté de faire bénéficier pour une part le Trésor public ». L'introduction de ces nouveaux types de dommages-intérêts est en tous points remarquable. 
Elle témoigne d'un certain esprit d'ouverture à l'égard de la common law et de l'impératif d'efficacité économique du droit. Ces variétés de dommages-intérêts ont bien une coloration économique, dans la mesure où elles sont susceptibles de procurer à la victime, un avantage économique que de simples dommages-intérêts compensatoires évalués par le juge ne lui apporteraient point. Raison pour laquelle ils n'ont pas encore acquis droit de cité dans notre droit positif, puisqu'ils se heurtent au principe cardinal de la réparation intégrale du préjudice, en vertu duquel les dommages-intérêts doivent réparer tout le préjudice, mais rien que le préjudice, et ne doivent donc pas constituer une source d'enrichissement pour la victime. Les textes comparés leur ouvrent cependant les portes de notre droit, dans une perspective morale assez marquée. En effet, dans certains domaines, tels la concurrence, la contrefaçon, la presse ou l'environnement, "l'alternative entre une condamnation civile purement indemnitaire et une condamnation pénale étroitement bridée par le principe de légalité des délits et des peines n'est pas satisfaisante car l'une comme l'autre laisse à l'auteur du dommage un très large avantage à continuer son activité nuisible et illicite $»^{22}$. Ainsi, face à l'inefficacité de la réparation civile et de la répression pénale à endiguer les fautes lucratives, les dommages-intérêts restitutoires et punitifs constituent un remède approprié en raison de leur caractère comminatoire, lequel assure le respect scrupuleux des règles légales et contractuelles.

24. Toujours au titre des convergences, il faut signaler une importante modification relative à l'indemnisation des victimes d'accidents de la circulation, dont les règles font désormais leur entrée dans le Code civil. En droit positif, pour des raisons politiques et économiques qui heurtent le sentiment le plus élémentaire de justice, le sort des victimes conductrices est très défavorable par rapport à celui que la loi de 1985 a réservé aux victimes non-conductrices. En effet, et en substance, alors que ces dernières sont toujours intégralement indemnisées de leurs préjudices corporels, sauf faute inexcusable, cause exclusive de l'accident à l'origine de leur dommage, ou faute intentionnelle, les victimes conductrices peuvent se voir opposer toutes leurs fautes et être privées de tout ou partie de l'indemnisation de leurs dommages corporels. Dans un souci particulièrement bienvenu de justice, les deux avant-projets traitent de façon identique toutes les victimes d'accidents de la circulation et mettent fin à la véritable discrimination qui défigurait la loi de 1985. Désormais, toutes les victimes de préjudices corporels subis à l'occasion d'un accident de la circulation sont soumises au même régime favorable.

22 G. Viney, eod. loc., sp. p. 68. 


\section{Bibliographie}

Rémy Ph. et Borghetti J.-S., "Présenta- Viney G., "Exposé des motifs", in tion du projet de réforme de la resAvant-projet de réforme du droit des ponsabilité délictuelle», in Pour une obligations et de la prescription, (dir.) réforme du droit de la responsabilité P. Catala, La documentation francivile, (dir.) F. Terré, Dalloz, 2011. çaise 2006. 